\title{
EL COMPORTAMIENTO REGIONAL DE LA INSERCIÓN LABORAL DE LOS MEXICANOS EN ESTADOS UNIDOS
}

\author{
SELENE GASPAR OLVERA, SIMDE, UAZ \\ RAFAEL LÓPEZ VEGA, CIEAP-UAEMEX
}

1

ntre los aspectos centrales

relativos al proceso social

migratorio de mexicanos

hacia la Unión Americana,

dada la estructura demográfica

todavía predominante de los

migrantes, destaca el de su

participación en los mercados

laborales en ese país. Los estudios

tradicionales al respecto pusieron

énfasis en la situación de autorización

para permanecer en Estados Unidos y

la ocupación por sector desempeñada.

Estudios de las dos últimas décadas se han centrado en el análisis de las

habilidades adquiridas en su calidad de inmigrantes que favorecen mejores puestos de trabajo y mejores salarios, como el lenguaje, y los valores de la cultura estadounidense. Parece natural que este tipo de estudios tengan como trasfondo o guía reflexiva la preocupación por la asimilación o integración social y cultural.

En los años noventa del siglo xx el estudio de la migración entre ambos países, ha generado un discurso en torno a la continuidad y cambio del patrón migratorio, discurso que en función de la investigación empírica suscita visiones un tanto polarizadas. Sin embargo, parecen predominar los siguientes nuevos elementos: el mayor origen urbano de los migrantes, la migración de familias completas, el mayor tiempo de permanencia en Estados Unidos, mayor presencia femenina, mejores niveles de escolaridad, la inserción en los mercados laborales estadounidenses de un mayor número de mexicanos en actividades del sector servicios o propiamente urbanas, la diversificación de los lugares de destino (Cornelius y Marcelli: 2000; Durand, Masey, Zenteno: 2001; Mendoza: 2003; Conapo: 2009).

La migración masiva reciente (tal vez hasta 2005-2006), originada por condiciones adversas en México, ha contribuido enormemente a la producción de situaciones «novedosas» para los migrantes; así frente a la heterogeneidad y diversificación, conceptos aparentemente neutrales, habría que leer también dispersión territorial y vulnerabilidad, expresadas en situaciones de pobreza, bajos 
salarios, en las numerosas muertes de mexicanos migrantes en la frontera estadounidense, en la necesidad de construcción de nuevos espacios, de nuevas comunidades en lugares donde la presencia de migrantes mexicanos tradicionalmente era escasa o nula, en los cuales se configuran y reconfiguran nuevas situaciones sociopolíticas, económicas y culturales que implican para la investigación cuantitativa y cualitativa una mejor exploración de la dispersión y vulnerabilidad de los mexicanos allende la frontera.

En este contexto, interesa aquí explorar a partir de la información de la Encuesta de la Comunidad Americana (ACS, ${ }^{1}$ por sus siglas en inglés) las características laborales y sociodemográficas de la población nacida en México que vive en los Estados Unidos, destacando la denominada heterogeneidad en los lugares de destino, su estudio a través de su agrupamiento por regiones de residencia o permanencia, los nexos de la ocupación y el ingreso con el nivel educativo, el dominio del idioma inglés, la antigüedad de la migración, entre otros elementos.

Dado que el presente trabajo en todo momento destaca la importancia

\footnotetext{
1 La Encuesta de la Comunidad Americana (ACS), por sus siglas en inglés, es una encuesta demográfica mensual y continua llevada a cabo por la oficina del Censo, que se tabula anualmente y a grandes rasgos replica el cuestionario ampliado del censo y su diseño muestral. De hecho, está previsto que esta última substituya al cuestionario ampliado del censo por lo que se convertirá en la principal fuente para el estudio de la población nativa y extranjera en Estados Unidos.
}

del factor regional en la ocupación, conviene como necesidad interpretativa hacer un distanciamiento frente a la concepción convencional de que los nuevos cauces seguidos en el asentamiento de los mexicanos al otro lado de la frontera, de que el cambio en el destino se liga preferentemente a nuevas oportunidades de empleo, mejores salarios o bien fallas de mercado en el país de origen que se ligan a estrategias deliberadas para evitar riesgos (Durand, Massey y Charvet 2000; Conapo: 2006; Donato y Bankston: 2008).

\section{ESTRUCTURA DEMOGRÁFICA ¿CUÁNTOS MEXICANOS RESIDEN EN ESTADOS UNIDOS?}

\section{Al Censo de Población y Vivienda} estadounidense del año 2000 le precede, por su importancia para estimar el volumen y estructura demográfica de los mexicanos residentes en Estados Unidos, la información que se deriva de la Encuesta Continúa de Población (CPS, ${ }^{2}$ por sus siglas en inglés), en la cual desde 1994 se aplica la pregunta sobre

2 La Current Population Survey (CPS) se ha levantado desde hace más de 50 años. Es una encuesta mensual de alrededor de 50,000 hogares a cargo de la oficina de censos para la oficina de estadísticas laborales, es considerada como la fuente primaria de información sobre las características de la fuerza laboral de la población de Estados Unidos y representa a la población civil no institucionalizada del país, entre sus principales limitaciones está el nivel de desagregación geográfico de análisis. 
el lugar de nacimiento de las personas que son residentes habituales en las viviendas de la dirección en muestra (usually lives at the adress) en territorio estadounidense. Después del Estudio Binacional (1996-1997), y de las cifras que en él se condensaron, éste se constituyó en el punto de referencia en torno a las estimaciones tanto del volumen como de la estructura demográfica de los mexicanos que habitualmente residen en Estados Unidos. Al respecto escasa o nula reflexión estadística se hacía en relación con el hecho de que las estimaciones derivadas de la CPS tenían a su vez como base los resultados de la enumeración censal estadounidense del año 1990.

La disponibilidad en 2003 de los resultados del censo del año 2000, como anticipan los propios documentos de la CPS (BLS: 2002: D-6, \$2), hacen que la encuesta tome a éstos como base para actualizar su diseño estadístico. A su vez los resultados del censo del año 2000 obligaron recientemente a una revisión y actualización de las proyecciones de población de ese país (Us Bureau of the Census: 2009), antes de estos resultados las proyecciones tenían como población base la estimada al primero de abril de 1990 (Hollmann, F. W., Mulder Kallan: 2000).

Conviene precisar que debido a que solamente en el cuestionario ampliado del censo estadounidense del año 2000 se inscribió la pregunta sobre lugar de nacimiento, no se cuenta con una enumeración actualizada (pese a las limitaciones que recabar información tiene de una población cuyo componente no autorizado ${ }^{3}$ roza el 60\%) de los mexicanos residentes en Estados Unidos, por lo que su estimación, según se deriva de los documentos técnicos que acompañan los resultados muestrales del censo, depende de su ajuste a las proyecciones de población.

En relación con la información censal estadounidense surgen también algunas diferencias de comparabilidad decenal; respecto a 1990 el censo de 2000 inscribe un notable cambio metodológico en la pregunta de apertura. Mientras que en 1990 se identifica una instrucción larga para que se anoten las personas que residen habitualmente en la vivienda, incluyendo a aquellas que declararon no tener una lugar fijo donde vivir, en este censo se tuvo especial cuidado en no contabilizar o incluir a la población que se encontraba de manera temporal y que tenía su residencia habitual en otro lugar (véanse los cuestionarios básico de 1990, y básico y ampliado de 2000 en http://www.census.gov). En 2000 la formulación es mucho más corta y explícita para que se liste a las personas que viven o se estaban quedando en la vivienda y hace especial énfasis en incluir a aquellas personas que se están quedando la mayor parte del tiempo mientras trabajan aunque tengan otro lugar donde vivir.

\footnotetext{
${ }^{3}$ De acuerdo con estimaciones del Pew Hispanic Center más del 55\% de los inmigrantes mexicanos son indocumentados y éstos representan cerca del $60 \%$ del total de indocumentados estimados en 11.9 millones (Mexican Inmigrant in the United State, 2008)
} 
A finales del siglo xx y en el inicio de este nuevo siglo empezó el levantamiento sistemático de la Encuesta de la Comunidad Americana (ACs, por sus siglas en inglés), que tiene como propósito principal sustituir el cuestionario censal ampliado. Esta encuesta vuelve a tener una nueva especificación para el concepto de residencia que guía el levantamiento, al considerar a las personas en su residencia actual (y no habitual), es decir incluye a todas aquellas personas que están viviendo o quedándose en la vivienda por más de dos meses (véase cuestionario de la ACs en http://www.census.gov).

Estas apreciaciones y otras diferencias metodológicas en los distintos instrumentos que se utilizan para obtener el volumen de inmigrantes en Estados Unidos y en particular de los mexicanos, repercuten de manera importante en su estimación debido a factores como, 1) la posible duplicidad de registros $y$ la captación de personas que en estricto sentido no residen en la vivienda o en los Estados Unidos, y 2) en una posible mayor estimación para ciertos grupos de población susceptibles de estar sólo temporalmente en Estados Unidos (Tuirán y Corona: 2008), y 3) la posible subestimación de ciertos grupos de población en especial los indocumentados (Lowell, Perdezini y Passel: 2008), entre otras.

Cabe hacer notar, que si bien ya para fines de los años noventa del siglo xx la literatura especializada daba cuenta de ciertos cambios selectivos en el patrón o patrones migratorios entre ambos países (véase Cornelius: 1992 y Cornelius y Marcelli: 2000; Canales: 1999), gran parte del diálogo experto está centrado en los ejes conceptuales de la propia dinámica migratoria, de éstos destaca la conceptualización de la migración como permanente, temporal y circular, con escasa reflexión o incorporación de criterios estrictamente demográficos que guían el levantamiento de la información utilizada en una buena parte de la investigación reciente (López Vega: 2007).

Las estimaciones sobre el volumen de mexicanos residentes en los Estados Unidos a partir de las fuentes disponibles apuntaban todavía hace tres años sin lugar a dudas hacia su incremento, hoy la información disponible muestra que su magnitud se ha estabilizado en alrededor de 12 millones de personas, población que representa $4 \%$ de la residente en Estados Unidos y 11\% de la residente en México (véase gráfica 1).

El análisis de la población mexicana radicada en Estados Unidos, quedaría incompleto si dejáramos de lado a los méxico-americanos ${ }^{4}$ que son los descendientes de mexicanos nacidos en nuestro país, población que actualmente asciende a 19 millones

\footnotetext{
${ }^{4}$ México-americanos: Población nacida en Estados Unidos que se declara de ascendencia u origen mexicano, o bien afirma ser hispano de origen mexicano, méxico-americano o chicano. La ascendencia se refiere a la nacionalidad o el país en el cual una persona o los padres o ancestro de la persona, nacieron antes de llegar a los Estados Unidos.
} 


\section{GRÁFICA 1}

Volumen de mexicanos en Estados Unidos, varios años

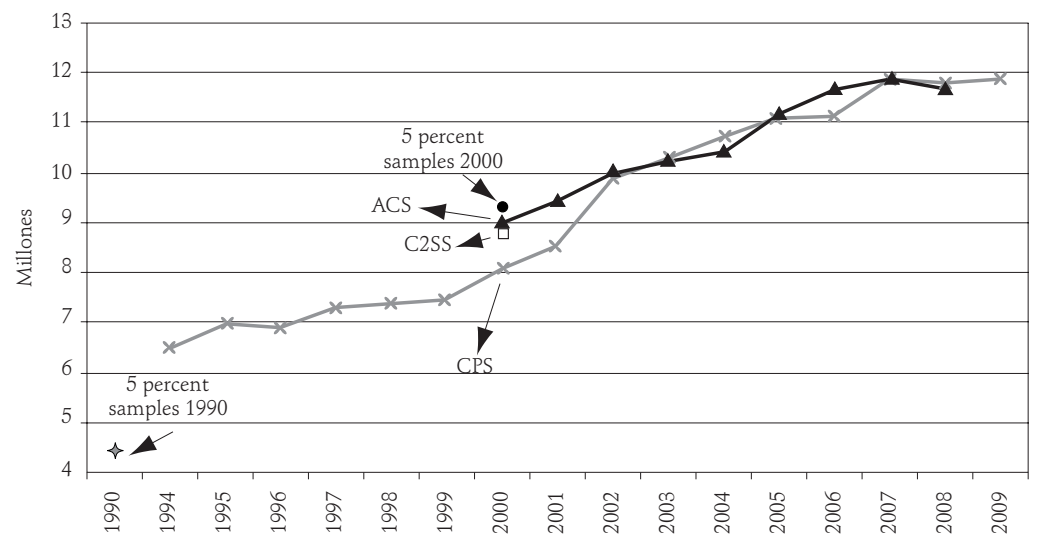

Fuente: SIMDE, UAZ, Estimaciones de los autores con base en US Bureau of the Census, 5-percent sample 2000. BLS, Current Population Survey (CPS), March Supplement 1994-2009, Supplementary Survey 2000 (C2ss), American Comunity Survey (ACS) 2000-2008

(6\% de la población total de Estados Unidos) que junto a los 12 millones de mexicanos de nacimiento suman ya 31 millones $(10.4 \%$ de la población total de Estados Unidos y $29 \%$ respecto de la población de México).

En relación con sus componentes demográficos, se observa que si bien hay todavía predominancia masculina $(55 \%)$ y en edades laborales, la brecha entre ambos sexos no es muy amplia. No obstante, se identifica también que en el proceso social migratorio hay claramente una participación de niños y la presencia de adultos mayores. Los méxico-americanos, a

\section{GRÁFICA 2}

Estructura demográfica de la población hispana (proyecciones) 2000, de la población nacida en México y de la población México-Americana residente en Estados Unidos, 2007

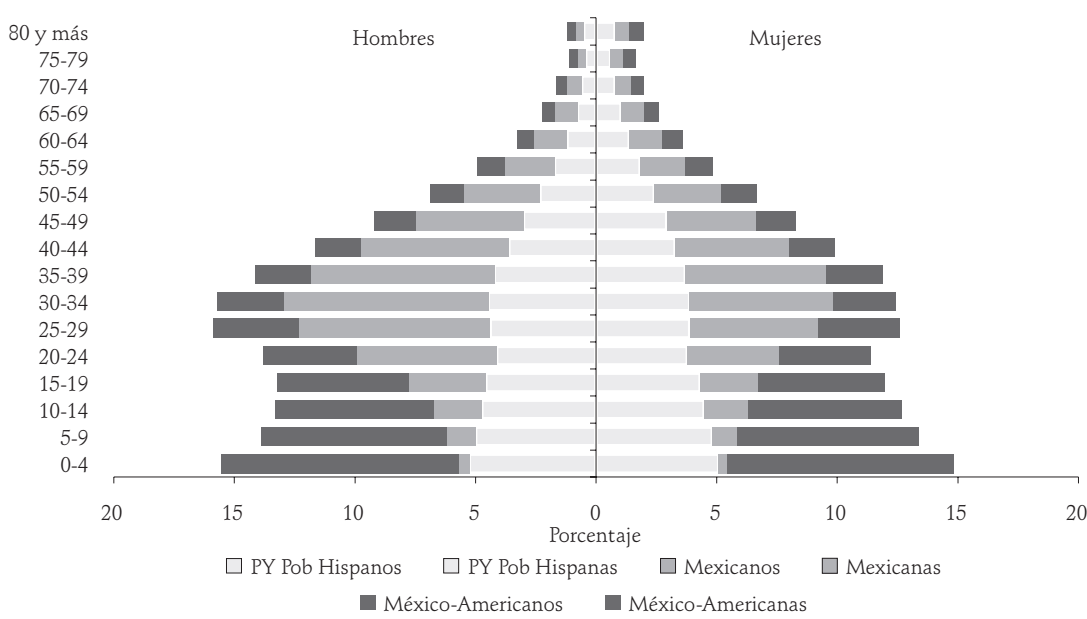

Fuente: SIMDE, UAZ. Elaboración de los autores con base en us Bureau of the Census, Proyecciones de Población de Estados Unidos, 1995-210; y estimaciones propias con base en American Community Survey (ACS), 2007. 
diferencia de sus contrapartes se conforman por una menor proporción de personas en edad laboral, (49\%), en tanto que los niños abarcan el $47 \%$, es decir 6 de cada diez descendientes de mexicanos tiene entre 0 y 14 años de edad, y cerca del $4 \%$ son adultos mayores, no existen diferencias significativas entre hombres y mujeres. Cada uno de estos componentes migratorios sin duda requiere ser analizado, contextualizado, delimitado. Por ahora, este estudio se limita a la exploración y análisis de los mexicanos residentes en Estados Unidos en función de su situación laboral en diferentes regiones de dicho país (véase gráfica 2).

\section{TRAZANDO TERRITORIOS. NUEVOS DESTINOS MIGRATORIOS DE LOS TRABAJADORES MEXICANOS EN ESTADOS UNIDOS}

En la literatura reciente, que describe la distribución territorial de los mexicanos en las ciudades, metrópolis, estados y regiones de Estados Unidos, se distinguen dos criterios: el destino ligado a la propia historicidad del fenómeno migratorio que inscribe a estados que han fungido tradicionalmente como el lugar de llegada y estancia, y de acceso a territorio estadounidense, y los nuevos lugares receptores o «emergentes» en los que la presencia de población nacida en México empieza a cobrar importancia.
A lo largo de casi todo el siglo pasado, los migrantes mexicanos en Estados Unidos se concentraron territorialmente en algunos estados, el comportamiento de la economía en las regiones de la Unión Americana es un elemento objetivo que influyó en el destino (Cf. AILF: 2002), la oferta de empleos y el propio perfil sociodemográfico de los migrantes son otros tantos factores que contribuyeron a forjar el destino migratorio. La propuesta de regionalización hecha por Durand y Massey $(2003)^{5}$ facilita la comprensión temporal de los asentamientos en los Estados Unidos; sin embargo, hoy todavía destacan los estados fronterizos, en ellos radican dos de cada cinco mexicanos (California, Texas, Nuevo México y Arizona). Es importante advertir que en los últimos 5 años, regiones como la Costa Este y la denominada Otra Región muestran un mayor crecimiento porcentual en relación con el resto de las regiones (véase mapa 1 y cuadro 1$)$.

5 Sudoeste Primera Fase: Arizona, California, Nuevo México y Texas. Sudoeste en Expansión: Idaho, Oregón, Nevada, Utah y Washington. Grandes Planicies: Illinois, Indiana, Michigan y Wisconsin. Costa Este: Carolina del Norte, Carolina del Sur, Connecticut, Delaware, Distrito de Colombia, Florida, Georgia, Maryland, Nueva Jersey, Nueva York, Pennsylvania, Rhode Island y Virginia. Grandes Planicies: Colorado, Iowa, Kansas, Missouri, Nebraska, Oklahoma y Wyoming. Otra Región: Alabama, Alaska, Arkansas, Dakota del Norte, Dakota del Sur, Hawaii, Kentucky, Louisiana, Maine, Massachusetts, Minnesota, Mississippi, Montana, Nueva Hampshire, Ohio, Tennessee, Vermont y Virginia Occidental. Grandes Lagos: Illinois, Indiana, Michigan y Wisconsin. 
MAPA 1

Crecimiento porcentual de la población de origen mexicano por región en Estados Unidos, 2003-2007

Nacidos en México

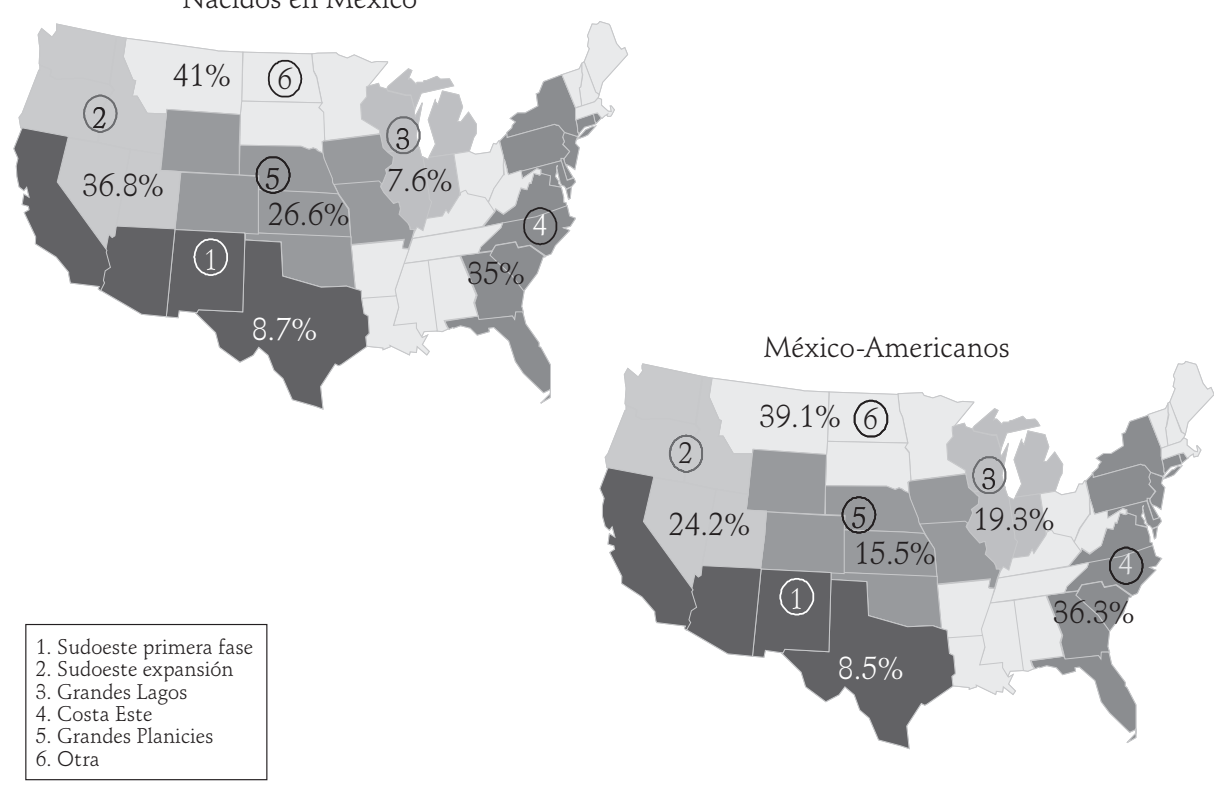

\begin{tabular}{|l|r|r|r|r|}
\hline & $\begin{array}{c}\text { DISTRIBUCIÓN DE } \\
\text { LA POBLACIÓN } \\
\text { NACIDA EN } \\
\text { MÉXICO }\end{array}$ & & \multicolumn{2}{|l|}{ 2003-2007 } \\
\hline & 2007 & TOTAL & MEXICANOS & MÉXICO-AMERICANOS \\
\hline Total & 100.0 & 9.7 & 10.7 & 9.1 \\
\hline Sudoeste Primera Fase & 67.4 & 8.6 & 8.7 & 8.5 \\
\hline California & 38.9 & 9.2 & 9.9 & 8.7 \\
\hline Texas & 21.4 & 6.3 & 3.1 & 7.9 \\
\hline Nuevo México & 1.2 & -3.6 & 2.4 & -5.9 \\
\hline Arizona & 5.8 & 19.4 & 25.1 & 16.1 \\
\hline Sudoeste Expansión & 6.6 & 29.4 & 36.8 & 24.2 \\
\hline Washington & 1.9 & 28.5 & 66.0 & 10.5 \\
\hline Resto de Sudoeste expansión & 4.7 & 29.7 & 27.9 & 31.2 \\
\hline Grandes Lagos & 8.5 & 14.1 & 7.6 & 19.3 \\
\hline Illinois & 6.2 & 11.7 & 3.4 & 19.8 \\
\hline Resto de Grandes lagos & 2.3 & 19.3 & 20.7 & 18.5 \\
\hline Costa Este & 12.6 & 35.6 & 35.0 & 36.3 \\
\hline Georgia & 2.4 & 22.8 & 14.9 & 35.3 \\
\hline Florida & 2.8 & 33.1 & 40.5 & 26.3 \\
\hline Resto de Costa Este & 7.5 & 41.3 & 40.9 & 41.7 \\
\hline Grandes Planicies & 4.8 & 19.3 & 26.6 & 15.5 \\
\hline Colorado & 2.1 & 7.2 & 10.1 & 5.8 \\
\hline Resto de Grandes planicies & 2.7 & 32.5 & 44.0 & 26.5 \\
\hline Otra & 3.3 & 39.8 & 41.0 & 39.1 \\
\hline
\end{tabular}

Nota: En 2007 se excluye a la población en viviendas colectivas para hacerlo comparable con 2003. Fuente: SIMDE, UAZ. Estimaciones de los autores con base en us Bureau of the Census, American Community Survey (ACS) 2003 y 2007. 


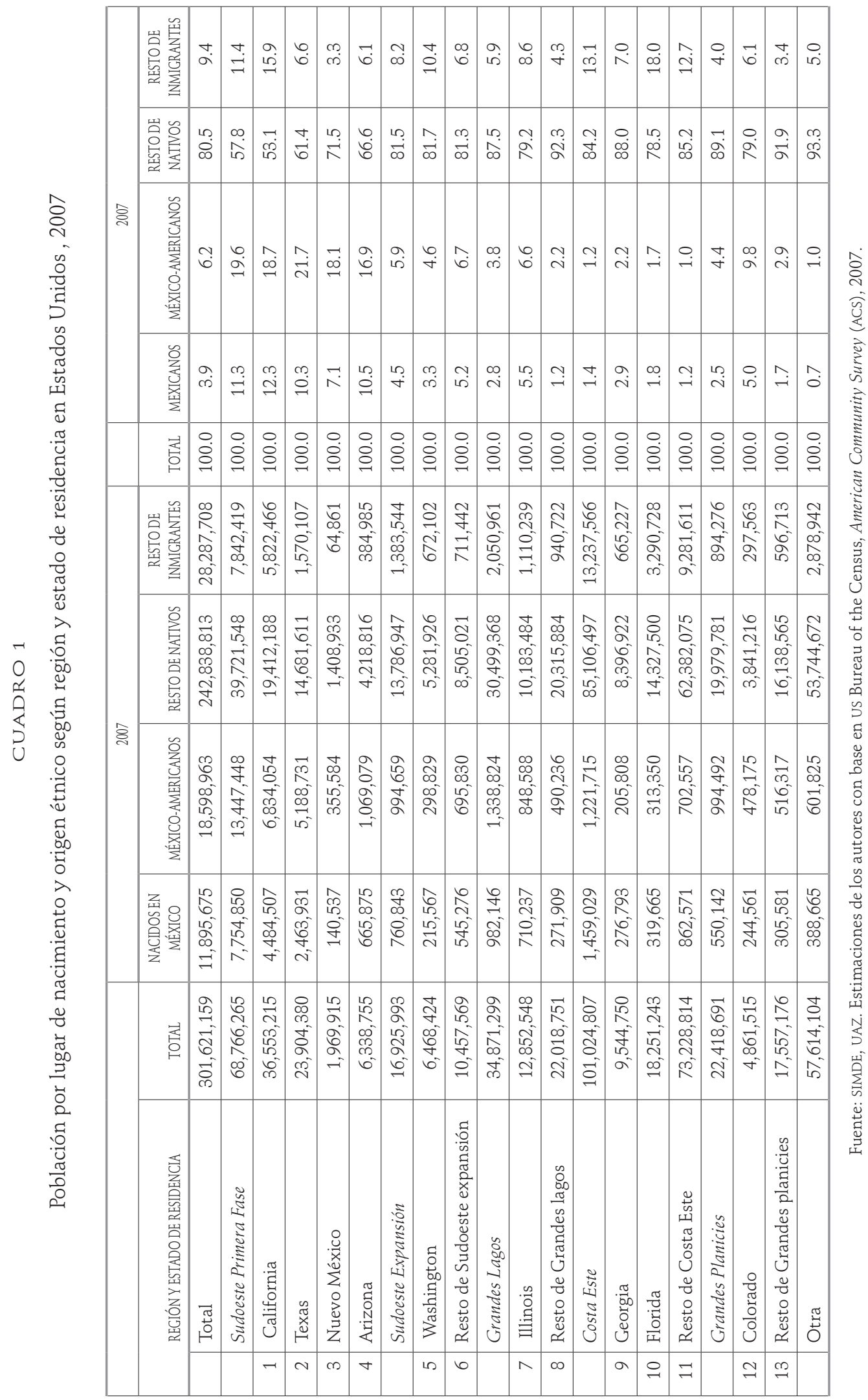


Históricamente los Grandes Lagos con Illinois, en primer lugar, fueron un espacio importante para la ocupación de mano de obra mexicana; en años recientes otros estados han cobrado importancia, es el caso de los ubicados en la denominada región Sudoeste Expansión, en ésta el trabajo y la ocupación se han estructurado en torno a la agroindustria y pizca de fruta. Al respecto debe tenerse en cuenta que el estado de Washington concentra alrededor del $57 \%$ de la producción de manzana en los Estados Unidos, y que como parte de esa capacidad productiva se definen y estructuran «nuevas» relaciones territoriales en la geografía económica, en este caso del oeste estadounidense (Jarosz y Qazi: 1999; Morales: 2001; Durand, Massey y Capoferro, 2005).

La configuración de territorios migratorios, marca como uno de sus componentes principales la demanda de fuerza de trabajo, por ejemplo el tipo de ocupaciones que han surgido en la región de la Costa Este, incluso en industrias viejas y con riesgos claros para la salud han favorecido patrones de migración temporal desde entidades de origen tradicionalmente no ligadas al fenómeno migratorio y movimientos internos de población (Benson: 1996; Griffith: 1997; Walker y Lawrence: 2004).

La notable reciente importancia de regiones y estados no vinculados tradicionalmente a la migración, y que hoy configura una distribución territorial de ésta que abarca prácticamente a todos los estados de la Unión Americana, plantea, en el contexto actual de estabilidad de su volumen, diferencias relativamente importantes al considerar a la población nacida en México en edades laborales respecto a la población ocupada, destacando el Sudoeste en expansión y la Costa Este (véase gráfica 3).

Este incremento positivo absoluto y relativo de la población nacida en México en edades laborales y ocupada en todas las regiones estadounidenses

GRÁFICA 3

Promedio de incremento porcentual anual 2003-2007, de la población en edades laborales y ocupada nacida en México, residente en Estados Unidos

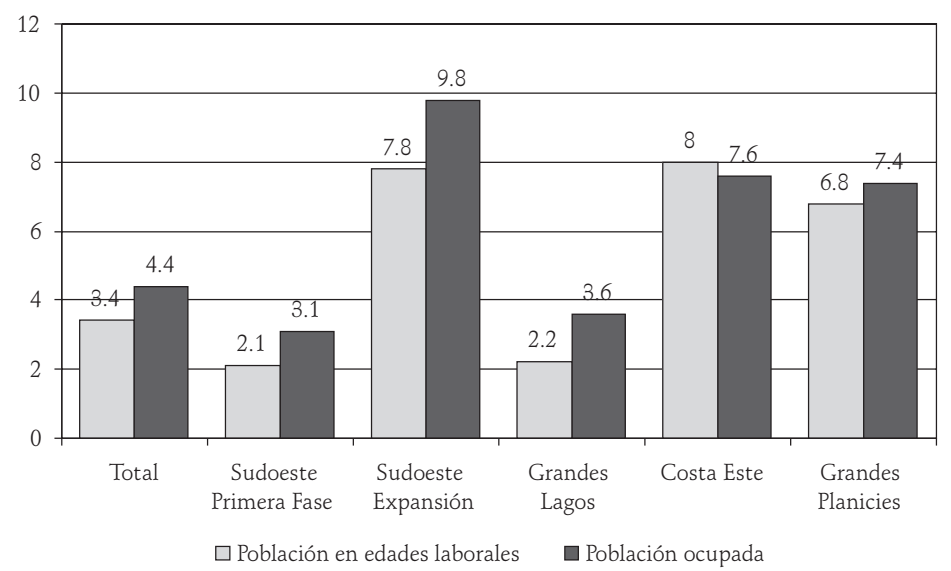

Fuente: SIMDE, UAZ. Estimaciones de los autores con base en US Bureau of the Census, American Community Survey, (ACS), 2007. 
implica considerar si los empleos a los que éstos han tenido acceso se mantienen en el campo de los sectores de actividad económica y ocupaciones específicas que para su estructuración contextualizan la presencia de los mexicanos como no autorizados, de niveles de escolaridad bajos, de escaso dominio de la lengua inglesa, y en consecuencia de bajos salarios, o bien existe una oferta de empleos que requieren ser ocupados por un perfil sociodemográfico acorde a ellos. Ya en 2002 se diagnosticó que en los próximos años (hasta 2010) alrededor de dos de cada cinco empleos nuevos en la economía estadounidense tendrían un requerimiento educativo mínimo (AILF: 2002).

\section{HACIA LA IMPOSICIÓN DE UN PERFIL LABORAL DE LOS MEXICANOS EN LA ECONOMÍA ESTADOUNIDENSE}

En torno a los inmigrantes mexicanos y sus ocupaciones, la narrativa reconoce la importante contribución de éstos a la economía estadounidense, y describe también que debido al bajo perfil educativo y lingüístico (dominio del inglés) de los inmigrantes mexicanos, las ocupaciones en las que se inscriben van acorde a este perfil (véase Giorguli y Gaspar: 2008).

No obstante del número de inmigrantes mexicanos de 25 años o más con escolaridad profesional y posgrado que se encuentran viviendo en Estados Unidos (medio millón aproximadamente), alrededor de $75 \%$ se encuentra trabajando y de éstos menos de la mitad logra insertarse en actividades acorde a su nivel de escolaridad (González: 2008; López y Villa: 2008; Conapo 2008), a nivel regional los trabajadores mexicanos con licenciatura y posgrado se encuentran principalmente en la región Sudoeste primera fase (65\%), seguida por la Costa Este con 12\% y Grandes Lagos (8\%).

Aunque en general se acepta que los puestos de trabajo a los que acceden los inmigrantes son empleos que los nativos o las personas con mayores niveles de educación no están dispuestos a desempeñar, se ha encontrado que los nativos son también un factor de competencia en los mismos mercados de trabajo. $\mathrm{Al}$ respecto, si comparamos los mexicanos con los nativos ${ }^{6}$ que no han concluido la secundaria (5.9 millones (53\%) contra 21.7 millones (13.6\%) respectivamente) se observa que $66 \%$ de los mexicanos y 38.5\% de los nativos están trabajando; y 29\% contra $57 \%$ son inactivos y los nativos con ese nivel de escolaridad ostentan tasas más altas de desempleo que los mexicanos (6 contra 17\%).

Como una aproximación a las diferencias en los perfiles de los mexicanos ocupados en las regiones analizadas, el cuadro 2 muestra algunos rasgos sociodemográficos que en otros estudios han resultado analíticamente útiles para explorar el mejor acceso a servicios de salud, empleo, ingresos y a eludir situaciones de pobreza propias de una sociedad en

\footnotetext{
${ }^{6}$ Excluye méxico-americanos.
} 


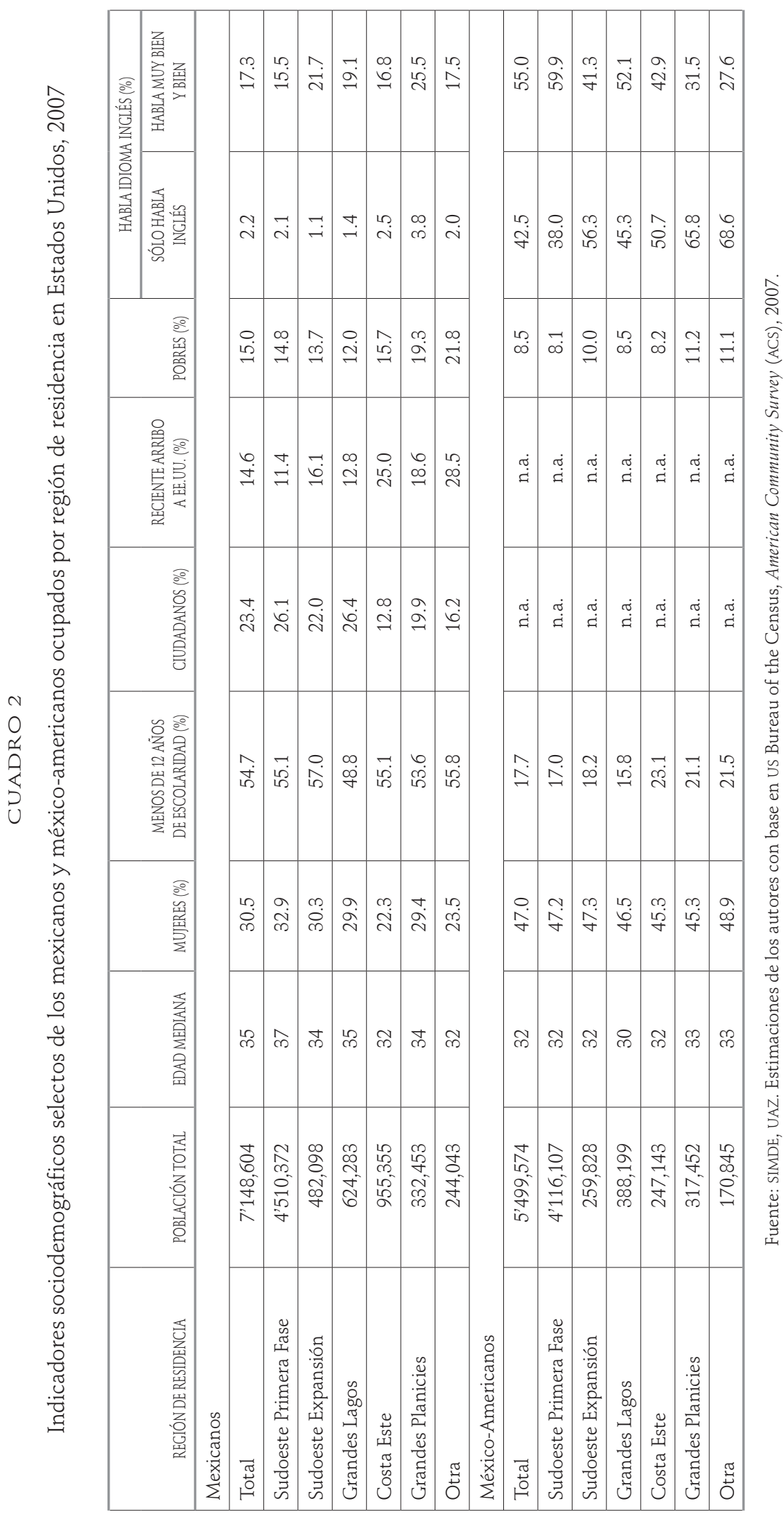


la que las personas enfrentan condiciones adversas de manera individual, es decir con sus propios recursos.

El desglose regional de los indicadores sociodemográficos seleccionados permite observar con claridad algunas relaciones y distinciones en el perfil de los mexicanos ocupados en la economía del vecino país del norte. Son múltiples los puntos que pueden destacarse, por ejemplo en los Grandes Lagos, Sudoeste Primera Fase y Sudoeste Expansión uno de cada cuatro mexicanos cuenta con la nacionalidad estadounidense, y muy pocos (uno de cada diez aproximadamente) entraron en los últimos diez años. Por su parte en la Costa Este uno de cuatro entraron recientemente a los Estados Unidos y uno de cada diez adquirió con la nacionalidad estadounidense. Sin embargo, llama la atención que en todas las regiones el nivel de escolaridad de los mexicanos sea menor a 12 años, es decir prevalece entre éstos un nivel educativo en general bajo.

En relación con la información sobre el dominio de la lengua inglesa, destaca que los porcentajes sobre: sólo habla inglés, van acorde, claro al hecho de que se trata de personas nacidas en México, y cuyo perfil educativo queda establecido con esos menos de doce años completos de escolaridad. Sin embargo, el hecho de que, salvo en las Grandes Planicies y en el Sudoeste Expansión, aproximadamente uno de cada cinco mexicanos ocupados respondan sobre que hablan muy bien o bien el inglés llama la atención dado que en general implica tener en cuenta la importancia que como cohorte migratoria tiene el aprendizaje de la lengua inglesa y de otras habilidades necesarias para el dominio de ésta, pues en general se ha planteado que en torno a los inmigrantes existen también mercados de trabajo en los que la lengua del país de origen es tan importante como el inglés (Carnevale, Fry y Lowell: 2001; Chiswick: 2009).

La ACs indaga sobre los ingresos percibidos durante los últimos doce meses, información que en sí misma resulta ya valiosa y que le permite al us Bureau of the Census establecer un índice de pobreza (Bishaw y Semega: 2008). En el cuadro 2 resulta alentador que los valores del estadístico individual de pobreza inscriban, salvo en las Grandes Planicies a menos de dos de cada diez mexicanos residentes en los Estados Unidos, y que el menor valor de personas en pobreza se presente en los Grandes Lagos, con $12 \%$.

A la luz de los enfoques de género en el proceso social migratorio se ha destacado cada vez la mayor presencia de las mujeres que van a trabajar a la economía estadounidense. Hasta ahora las mujeres han seguido el cauce masculino en las regiones estadounidenses donde se ocupan. Más de dos terceras partes se ubican en la región Sudoeste Primera Fase, aunque es necesario reconocer que su destino, al igual que el de los hombres, también presenta elementos de dispersión en el momento actual, sin embargo, con volúmenes apenas inferiores al de los hombres en todas las regiones. 
GRÁFICA 4

Población económicamente activa nacida en México y su tasa de ocupación por región de residencia en Estados Unidos, según condición de migración y sexo, 2007

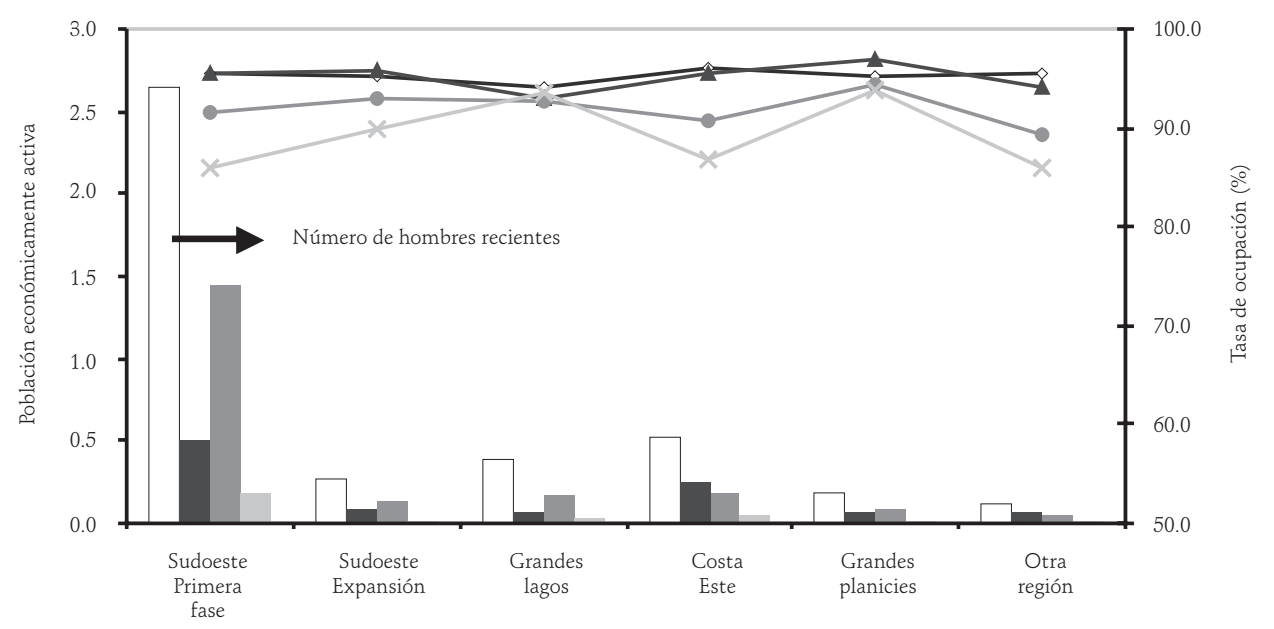

Fuente: SIMDE, UAZ. Estimaciones de los autores con base en Us Bureau of the Census, American Community Survey (ACS), 2007.

Es posible que las diferencias regionales se estructuren en términos de una migración tradicional masculina que ha arrastrado con ella a mujeres y niños a la región Sudoeste Primera Fase, pero también a una migración reciente con integrantes más jóvenes hombres y mujeres que se establecen en nuevas regiones, en volumen y con tasas de ocupación distintas, nuevas regiones en las cuales no ser migrante varón podría resultar relevante para acceder al empleo, y aún más si se es mujer y se ingresó recientemente a la Unión Americana (véase gráfica 4).

\section{SECTORES DE ACTIVIDAD Y OCUPACIONES ESPECÍFICAS DE LOS MEXICANOS EN LA ECONOMÍA ESTADOUNIDENSE}

Los mercados de trabajo en los que se ocupa a la mano de obra mexicana que reside en Estados Unidos responden, como es natural en buena medida a la propia lógica de las condiciones estructurales que condicionan la existencia de empleos, y claro a la oferta misma de mano de obra. La concentración y dispersión de los mexicanos en territorio estadounidense los hace visibles en prácticamente todas las regiones de ese país, pero también los hace visibles en todos los sectores de actividad económica.

No debe pasar inadvertido que, a pesar de la argumentación esgrimida en algunas investigaciones de que en la Unión Americana predominan las actividades en servicios y menores niveles de calificación, y que sólo son de destacar algunas diferencias regionales, tanto información de CPS como de la ACS, así como encuestas del Pew Hispanic Center muestran que hay una diferenciación regional en las actividades económicas de ocupación 


\section{GRÁFICA 5}

Principales sectores industriales en los que se ocupan los mexicanos

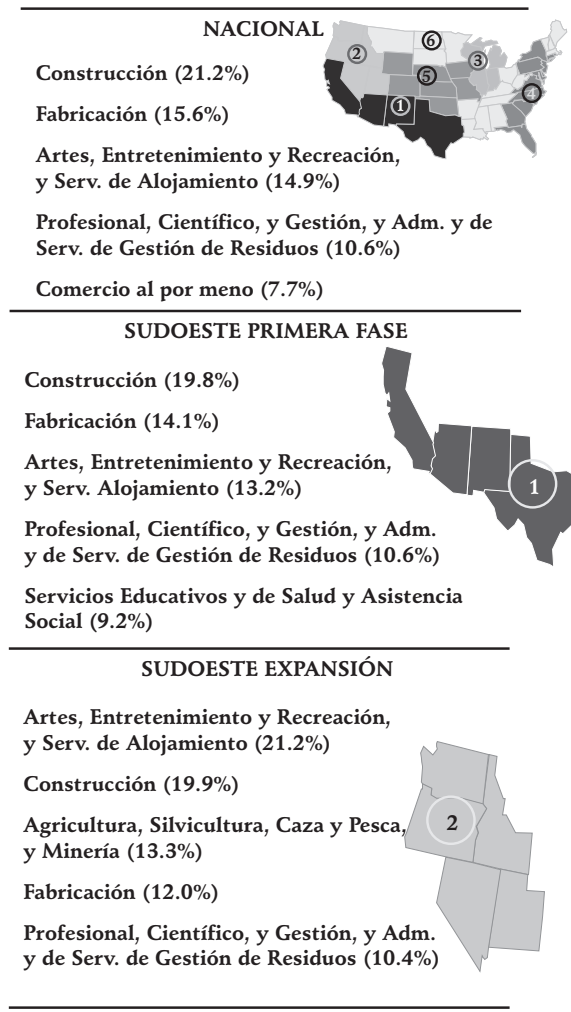

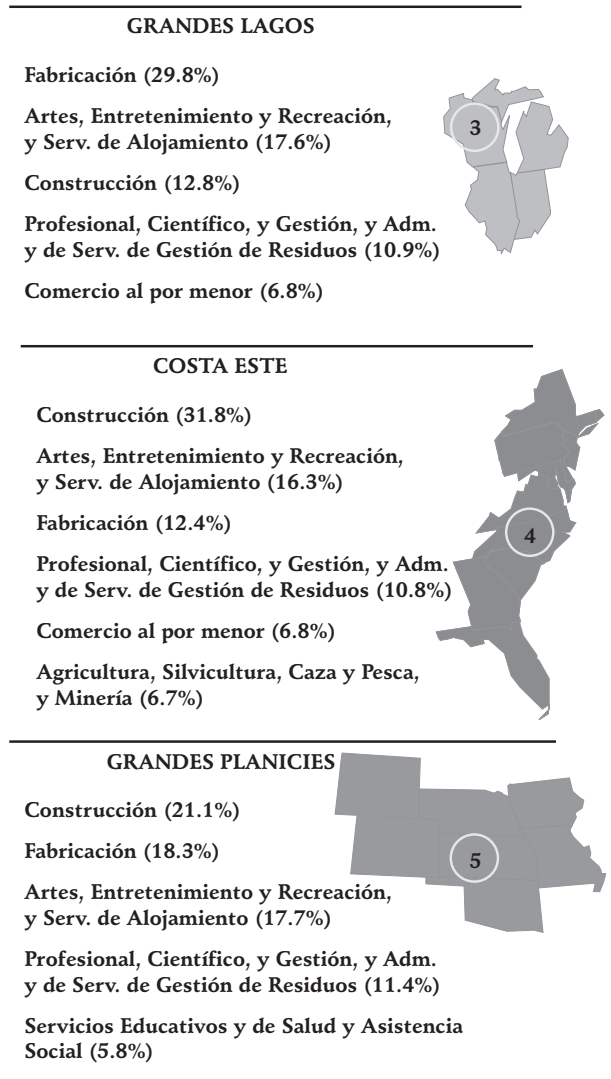

Fuente: SIMDE, UAZ. Estimaciones de los autores con base en us Bureau of the Census, American Community Survey (ACS), 2007.

de los mexicanos en Estados Unidos (Kochhar: 2005). Tal vez los grandes números encubren procesos específicos de demanda de mano de obra. Por ejemplo, su inserción en actividades económicas de vieja data y en las que en general se reconoce que los trabajadores requieren poca calificación (pero en los que la experiencia es relevante) como el de la construcción (Costa Este), o bien en actividades de manufactura y comercio (Grandes Lagos) es en el siglo XXI de importancia permanente.

Las ocupaciones a las que los mexicanos acceden en Estados Unidos responden en primer lugar a la oferta de puestos de trabajo, al perfil laboral (habilidades, capacidades, experiencia, educación) requerido en éstos, a las características sociodemográficas de los mexicanos y a su situación jurídica en dicho país. La distribución por ocupaciones muestra variaciones muy pequeñas entre regiones. En la Costa Este los trabajadores de la construcción son ligeramente predominantes, mientras que en la región de los Grandes Lagos quienes trabajan como obreros y trabajadores especializados son claramente predominantes (véase grafica 5 y cuadro 4). 


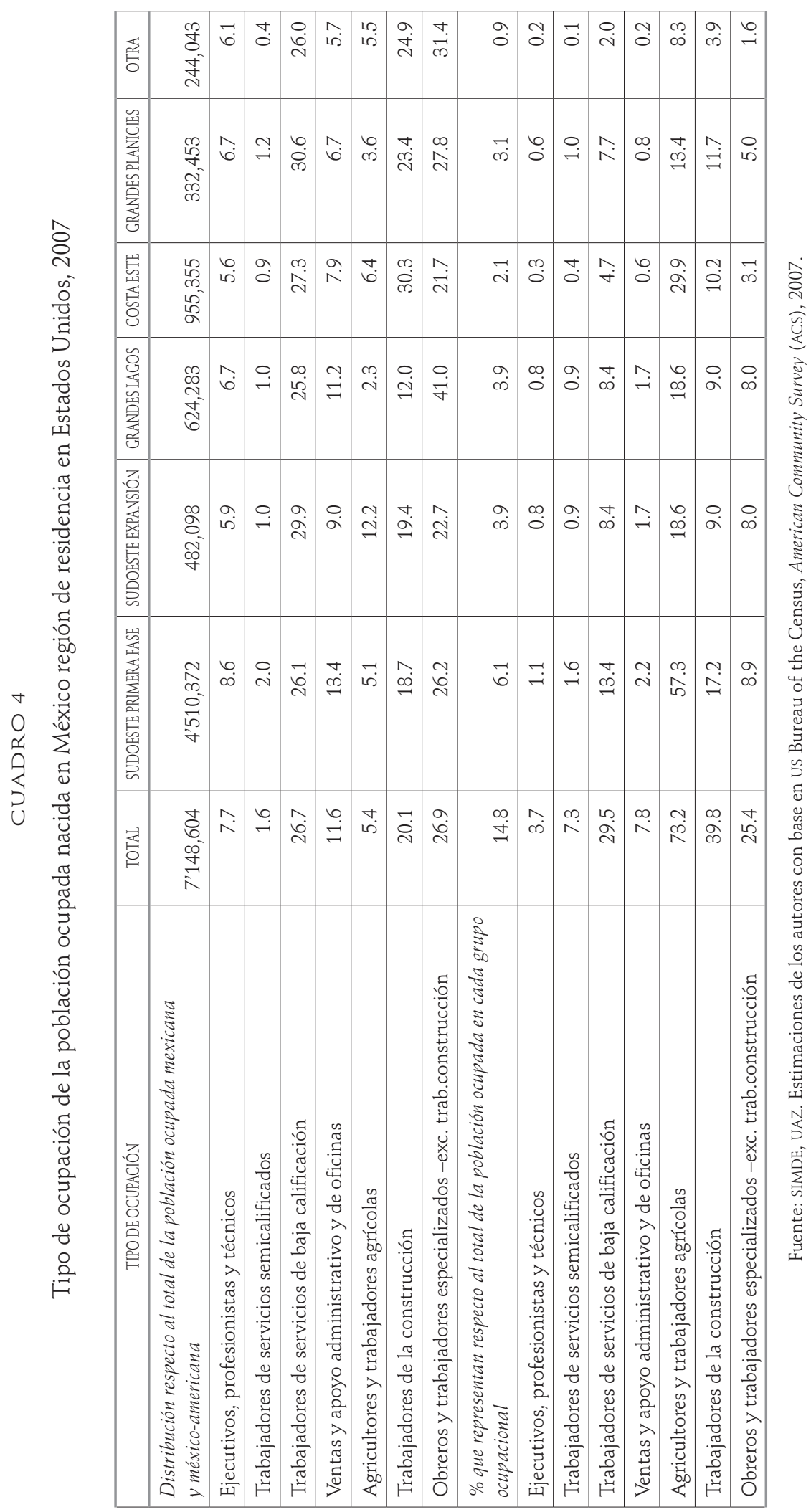




\section{GRÁFICA 6}

Ingreso promedio anual (dólares) de los trabajadores mexicanos en Estados Unidos por características seleccionadas, 2007

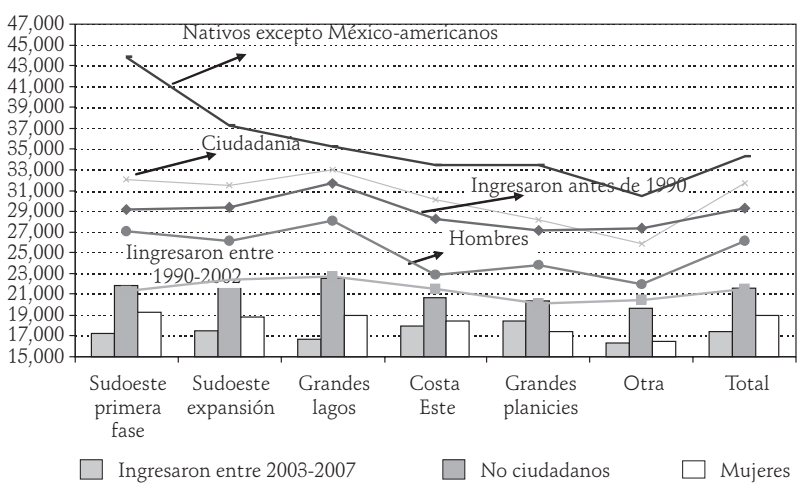

Fuente: SIMDE, UAZ. Estimaciones de los autores con base en us Bureau of the Census, American Community Survey (ACS), 2007.

Los estudios económicos y sociológicos llevados a cabo desde aproximadamente dos décadas en relación con los ingresos percibidos por los mexicanos inmigrantes en los Estados Unidos y la presión que la presencia de mano de obra mexicana tiene sobre los mercados laborales locales y los ingresos de la población local, probaron, indica Chiswick (2009), desde entonces, que los inmigrantes tienen escaso o nulo efecto sobre los salarios de los trabajadores nativos. Sin embargo, todavía es posible encontrar estudios basados en información actualizada en los que se destacan los impactos negativos efectivos de la migración sobre los mercados de trabajo locales, en particular en lo que se refiere a empleos y salarios (Peri: 2007).

La construcción social del conocimiento en torno a la importancia e impacto de los inmigrantes en la economía y sociedad estadounidense, está marcada por situaciones que giran en torno a la percepción y valoración social de los inmigrantes, lo cual adquiere dimensiones distintas en la divergente geografía demográfica y política estadounidense, pero sin duda, pese a la convergencia metodológica de distintos autores (nativos versus inmigrantes), también a la especificidad de los modelos, variables seleccionadas, periodo de cobertura y calidad de los insumos utilizados (Borjas: 2003; Gianmarco y Peri: 2006; NCLR: 2008). La gráfica 5 muestra el ingreso promedio anual (en dólares) percibidos por los mexicanos ocupados en la economía estadounidense; se hace visible cómo la ciudadanía, antigüedad migratoria, condición masculina, condicionan la percepción de mayores ingresos. A escala regional, en los Grandes Lagos, para cualquiera de las características mencionadas de los mexicanos, se estiman los ingresos promedio más altos, mientras que en la Costa Este y las Grandes Planicies se observa una situación no tan favorable. 
Conceptualizar y estimar a la

migración internacional es un reto que se inscribe en la lógica del análisis demográfico, y en cuanto tal requiere ser abordado con los instrumentos analíticos que dotan a la información de precisión y calidad. Acudir al concepto de residencia habitual para introducirse en la reflexión sobre el fenómeno migratorio tiene como objetivo reflexionar sobre la pertinencia actual de continuar la reflexión sobre la migración internacional a la luz de categorías tradicionales, bajo la premisa de que se trata de un fenómeno demográfico, de un proceso social, de un hecho social total siempre dinámico cambiante, difícil de asir, el cual trastoca la vida cotidiana individual y familiar.

La movilidad territorial de los mexicanos fuera de las fronteras del país, por motivo de emigración, cuestiona las bases económicas, sociales y políticas que el Estado mexicano tiene para ofrecer a su población opciones de vida fundadas en una visión de largo plazo y en políticas públicas orientadas al bienestar social, y al mejor aprovechamiento del potencial que el crecimiento demográfico, y en particular que el fuerte componente joven ofrece. Para las autoridades y sociedad estadounidense los inmigrantes laborales mexicanos y sus familias, plantea a escala nacional y para muchos gobiernos locales, retos y oportunidades únicas en materia económica, social, política y cultural.
BLS (2002). Current Population Survey

Design and Methodology. Technical Paper 63RV, U.S. Department of Commerce Economics and Statistics Administration.

Cornelius, Wayne y Enrico Marcelli (2000), «The Changing Profile of Mexican Migrants to the United States: New Evidence from California and Mexico", IZA Discussion Paper Series, November. www.iza.org DonATO y Bankston (2008), «The origins of employer demand for immigrants in a new destination: the saliente of soft skills in a volatile economy», en D. Massey (ed.), New Faces in new places. The Changing Geography of American Immigration.

Durand, Douglas, S. Massey y Fernando Charvet (2000), "The Changing Geography of Mexican Inmigration to the United States: 1910-1996», Social Science Quarterly, vol. 81, núm. 1, marzo.

y Douglas S. Massey (2003), Clandestinos. Migración mexicana en los albores del siglo XXI, México, Editorial Miguel Ángel Porrúa. y Chiara Capoferro (2005), «The New Geography of Mexican Immigration in the United States», en Víctor Zúñiga y Rubén Hernández (eds.), New Destinations. Mexican Immigration in the United States, New York, Rousell Sage Foundation. y René M. Zenteno (2001), "Mexican Immigration to the United States: Continuities and Changes», Latin American Research Review, vol. 36, núm. 1.

Giorguli, Silvia E. y Selene Gaspar (2008), Inserción ocupacional, ingreso y 
prestaciones de los migrantes mexicanos en Estados Unidos, México, Conapo.

López Vega (2007), «Notas sobre migración internacional», presentación en la

Reunión Interinstitucional sobre la Conciliación Censal 2005-2006 llevada a cabo en Cuernavaca, Morelos, Julio.

Lowell, B. Lindsay, Carla Perdezini y Jefrey S. Passel (2008), La demografía de la migración México a Estados Unidos. La gestión de la migración México-Estados Unidos: Un enfoque Binacional. y Jesús Villa Cordero (2008), «Una aproximación a las implicaciones globales de la migración especializada: cifras para la dimisión de México», documento presentado en el 20 . Congreso Internacional Migraciones Globales Experiencias regionales y enseñanzas para México, abril, Mazatlán, Sinaloa.

Tuirán y Corona (2008), «Magnitud de la emigración de mexicanos a Estados Unidos después del año 2000», Papeles de población, núm. 57, julio-sep, Toluca, México, UAEM. 